\title{
LGE in pulmonary hypertension predicts clinical events
}

\author{
Siva Soma ${ }^{1 *}$, Srinivas Murali ${ }^{1}$, Raymond Benza', June A Yamrozik ${ }^{1}$, Ronald B Williams ${ }^{1}$, Mark Doyle', Diane Vido ${ }^{1}$, \\ Robert WW Biederman", Vikas Rathi \\ From 2011 SCMR/Euro CMR Joint Scientific Sessions \\ Nice, France. 3-6 February 2011
}

\section{Introduction \\ Introduction}

Right ventricular (RV) function predicts prognosis in pulmonary hypertension (PH) patients (pts) and Right Ventricular failure (RV). Prior studies evaluating of 3D RV ejection fraction (EF) have yielded inconsistent prognostic information. Here we explore the prognostic value of contrast induced CMR in PH patients with RV dysfunction.

\section{Purpose}

Hypothesis

We hypothesize that myocardial Late Gadolinium Enhancement (LGE) which a marker for myocardial fibrosis when present in RV or RV insertion points (RVIP) is a predictor of poor prognosis in $\mathrm{PH}$ pts.

\section{Methods}

A retrospective chart review of PH pts $(n=21)$ who underwent clinically indicated CMR were analyzed. Demographic data showed mean age 61 yrs; $28 \%$ male; $43 \%$ WHO group I, $29 \%$ group II, $14 \%$ group IV,14\% group V. RV volumetric data were indexed to BSA, and along with RVIP LGE information were correlated with major adverse clinical events (MACE) such as hospitalization, death and referral/need for lung transplantation.

\section{Results}

LGE was positive (+) in 14 pts (66\%) and (-) in the remaining 7 pts (33\%). Compared to LGE (- ) pts, the LGE (+) pts had higher RVEDVI, RVESVI and lower RVEF $(\mathrm{p}<0.05)$. However, only LGE in combination with 3D RVEF predicted MACE. Specifically, LGE (+) pts had 9 MCE compared to
1 in the LGE (-) pts. The median RVEF value was $45 \%$. The event rate was $89 \%$ in pts with LGE $(+)$ and RVEF $<45$ compared to $20 \%$ in LGE (-) and RVEF $<45 \%$. Group comparisons were done using the Fisher's exact test. The comparisons were not statistically significant due to small sample size.

\section{Conclusions}

Late Gadolinium Enhancement may be the pathophysiologic hallmark in patients with $\mathrm{PH}$ patients as a direct reflection of the underlying RV failure due to progressive myocardial fibrosis. Late gadolinium enhancement in combination with RVEF provides important prognostic information that accurately predicts important adverse $\mathrm{CV}$ outcomes such as lung transplantation and death. Prospective studies are needed to confirm this observation.

\section{Author details}

${ }^{1}$ Allegheny General Hospital, Pittsburgh, PA, USA. ${ }^{2}$ Bon Secours Heart and Vascular Institute, Richmond, VA, USA.

Published: 2 February 2011

doi:10.1186/1532-429X-13-S1-P297

Cite this article as: Soma et al:: LGE in pulmonary hypertension predicts clinical events. Journal of Cardiovascular Magnetic Resonance 201113 (Suppl 1):P297.

${ }^{1}$ Allegheny General Hospital, Pittsburgh, PA, USA

Full list of author information is available at the end of the article

(c) 2011 Soma et al; licensee BioMed Central Ltd. This is an open access article distributed under the terms of the Creative Commons 